\title{
IMPACTOS DO PROCESSO DE RECONHECIMENTO, VALIDAÇÃO E \\ CERTIFICAÇÃO DE COMPETÊNCIAS NO ALENTEJO (2001-2005): \\ A DIMENSÃO ESCOLAR
}

\author{
Bravo Nico (1), Lurdes Pratas Nico (1,2) \& Fátima Ferreira (1) \\ (1) Universidade de Évora (Centro de Investigação em Educação e Psicologia) / Portugal \\ (2) Direcção Regional de Educação do Alentejo/Portugal \\ jbn@uevora.pt
}

\section{Resumo}

No âmbito de um projecto de investigação, actualmente em curso no Centro de Investigação em Educação e Psicologia da Universidade de Évora ${ }^{1}$, definiu-se, como objectivo geral do estudo, conhecer e caracterizar os eventuais percursos escolares, profissionais e pessoais que os indivíduos certificados pelo processo de Reconhecimento, Validação e Certificação de Competências (RVCC), no Alentejo, no período 2001-2005, concretizaram após a conclusão desse processo.

No período considerado, existiam, no Alentejo, 6 Centros de RVCC em funcionamento (actuais Centros Novas Oportunidades) e foram certificados 2969 indivíduos.

$\mathrm{Na}$ presente comunicação, apresentar-se-ão alguns dos indicadores existentes que concorrem para a caracterização dos impactos registados, ao nível da dimensão escolar dos indivíduos que concretizaram o processo de RVCC (Nico, 2009).

\section{Introdução}

No ano de 2003, no Alentejo, foram certificados 751 (setecentos e cinquenta e um) indivíduos, os quais viram alteradas as respectivas habilitações académicas, através da concretização do processo de RVCC (Reconhecimento, Validação e Certificação de Competências) em quatro instituições promotoras de Centros de RVCC: Fundação Alentejo/Évora, ESDIME (Agência para o Desenvolvimento Local no Alentejo Sudoeste/Messejana), Centro de Formação Profissional de Portalegre do Instituto do Emprego e Formação Profissional e ADL (Associação de Desenvolvimento do Litoral Alentejano/Santiago do Cacém) que, na época, constituam a rede a operar no território daquela região do sul de Portugal.

Esta população foi submetida a um inquérito que assumiu, como objectivo, a avaliação de eventuais impactos da frequência deste percurso de qualificação e da obtenção da respectiva certificação, nas várias dimensões dos indivíduos: pessoal, profissional, social e continuação de trajectórias formativas.

\footnotetext{
${ }^{1}$ Projecto de investigação denominado "Novas Núpcias da Qualificação no Alentejo", promovido pelo Centro de Investigação em Educação e Psicologia da Universidade de Évora e financiado pela Fundação para a Ciência e a Tecnologia ( ref $^{\mathrm{a}}$ PTDC/CPE-CED/104072/2008).
} 
Os resultados obtidos, através das respostas recolhidas, evidenciam consequências pouco significativas nos percursos profissionais dos indivíduos, em nítido contraste com as mais evidentes alterações nos contextos pessoais e sociais.

$\mathrm{Na}$ presente comunicação, apresentamos os resultados parcelares obtidos através da aplicação de um inquérito a adultos certificados em processos de Reconhecimento, Validação e Certificação de Competências (RVCC) realizados, em 2003, em Centros de RVCC do Alentejo (Nico, 2009).

\section{Breve revisão da literatura}

É hoje um imperativo social a valorização dos conhecimentos e competências adquiridas através dos adquiridos experienciais, também como uma consequência da abertura da escola à sociedade civil e do reconhecimento de que a aprendizagem dos indivíduos se realiza para além da escola (Trigo, 2002). Esse imperativo tem tido uma afirmação crescente, quer no contexto internacional, quer no contexto nacional.

A este propósito destacam-se o papel de organismos como a UNESCO, as estratégias de Aprendizagem ao Longo da Vida (ALV) esboçadas em Declarações como a de Copenhaga e Bolonha, o Inventário Europeu de abordagens de validação impulsionado pela Comissão Europeia e pelo Centro Europeu para o Desenvolvimento da Formação Profissional (CEDEFOP) e Programas Europeus como o Grundtvig, por exemplo. Além de uma possibilidade de reconhecer e validar as aprendizagens adquiridas ao longo da vida, pretende-se o reforço de uma formação contínua que permita aos indivíduos continuar a aprender. Nem todos os países desenvolveram esses dispositivos de igual modo, o que se evidencia, tanto nos objectivos, como nos destinatários e metodologias que são utilizadas (Pires, 2005).

Em Portugal, relevamos as Conferências Europeias de 2000 (ANEFA, 2001) e 2007, em Évora e Lisboa, respectivamente, nas quais a preocupação fundamental assentou no reconhecimento e validação das aprendizagens formais e não formais. Outras iniciativas europeias vêm dar corpo a este paradigma de AL: i) a criação do Quadro Europeu de Qualificações (QEQ), lançado em 2007, naquela última conferência referida; ii) o Sistema de Créditos para a Educação e Formação Profissional (ECVET), que vem reforçar a transferência, capitalização e reconhecimento das aprendizagens adquiridas ao longo da vida, numa perspectiva de cooperação e confiança mútua entre os países europeus.

No Relatório produzido para a UNESCO, pelo Comité Internacional sobre Educação para o século XXI, (conhecido como o documento Educação: um tesouro a descobrir (Delors et $a l, 1996)$, sublinhou-se a importância do processo de reavaliação do conceito de competências para a vida ou competências-chave, no sentido de permitir ao indivíduo participar, activa e criticamente, na vida social e ampliar sua capacidade de inserção e mobilidade no mercado de trabalho (Alonso et al, 2001).

A origem de um sistema público e generalizado de Reconhecimento, Validação e Certificação de Competências (RVCC) foi inscrito, desta forma, na Estratégia Europeia para o 
Emprego e, em Portugal, através da implementação do Plano Nacional de Emprego. O surgimento desta modalidade de ensino tem as suas raízes em tempos distantes, principalmente na segunda metade do século XX, quando nos EUA, a desmobilização e a consequente entrada no mercado de trabalho de muitas centenas de milhares de soldados, que haviam participado da segunda guerra mundial, induziu a necessidade de promover a certificação das suas competências adquiridas em contextos não formais.

Mais tarde, na década de 60, no Québec (Canadá), assiste-se às lutas e reivindicações de grupos feministas no acesso ao Ensino Superior, sendo necessário criar dispositivos de qualificação, reconhecimento e validação dos adquiridos experiencias. No Reino Unido, por exemplo, assiste-se à implementação das Faculdades de Rede Aberta (OCN). O mesmo aconteceu na França, nos anos 80 (mais no sector produtivo), no México, no Chile (com uma gestão tripartida entre o Estado, os empregadores e os sindicatos) e no Brasil em 2000 com a implementação de sistemas de reconhecimento, validação e certificação de competências.

Esta possibilidade formal de reconhecimento, validação e certificação de competências adquiridas pelos indivíduos, em contextos não educacionais de aprendizagem, tem a sua origem em Portugal, com a criação da Agência Nacional para a Educação e Formação de Adultos/ ANEFA (1999-2002). A ANEFA foi um instituto público de dupla tutela (Ministério da Educação e Ministério da Trabalho e da Solidariedade Social) com a missão de, entre outras, construir um sistema de reconhecimento, validação e certificação de aprendizagens não formais dos adultos, visando sua certificação escolar e profissional e qualificação.

Em 2001, os Centros de RVCC (Centros de Reconhecimento, Validação e Certificação de Competências) são criados. Esses centros - actualmente designados Centro Novas Oportunidades - são entendidos como portas de reentrada no sistema de educação formal para aqueles que precisam de obter uma resposta de qualificação e são dirigidos a adultos (após os 18 anos) que desejam obter um equivalente certificação escolar e profissional para o Ensino Básico e Secundário.

Entre as várias atribuições dos $\mathrm{CNO}$, destacam-se três: (i) o acolhimento, diagnóstico e encaminhamento dos adultos que se queiram qualificar, através de uma das respostas inscritas no Sistema Nacional de Qualificações (Decreto-Lei n. ${ }^{\circ}$ 396/2007, de 31 de Dezembro); (ii) a realização de processos de RVCC, de nível básico ou secundário, quando o encaminhamento anterior é orientado para a necessidade de reconhecer e validar os adquiridos experiencias dos adultos adquiridos ao longo da vida, através da experiência de vida pessoal e profissional; (iii) a provedoria, traduzido no apoio ao adulto no período pós-certificação, através da elaboração do Plano de Desenvolvimento Pessoal (Gomes \& Simões, 2007:20-21).

Nesta nova abordagem à educação e formação de adultos, as Competências-chave para a Educação e Formação de Adultos (http://www.novasoportunidades.gov.pt.aspx) acabam por valorizar a importância das experiências de aprendizagem não formal concretizada em ambientes de educação (Alonso, et al 2001). 
No âmbito do processo de Reconhecimento, Validação e Certificação de Competências, os adultos são confrontados com a utilização de uma metodologia das Histórias de Vida, "recordando" e reflectindo sobre todas as situações que lhes possam ter proporcionado aprendizagens e competências significativas. Assim, são confrontados com uma multiplicidade de contextos de aprendizagem, com o contributo de diversas instituições formais e não formais que diferem entre si, quer nos espaços, quer nas metodologias ou intencionalidade da aprendizagem que aí se concretiza. Partindo daí, os adultos constroem o Portefólio Individual de Aprendizagens que assume duas dimensões principais (Correia, 2006): i) uma retrospectiva (reconhecimento pessoal e dos outros, auto-avaliação); ii) outra, prospectiva, de reconhecimento do potencial de aprendizagem adquirido e a adquirir. No decorrer deste processo, há um potencial educativo que se revela, embora não se tenha, na maior parte das vezes, consciência disso.

Desde a criação do Sistema Nacional de RVCC (2001) que, em Portugal, muitos adultos viram certificadas as competências adquiridas na redes formais e não formais de aprendizagem. Actualmente, o dispositivo de RVCC desenvolve-se numa rede de Centros, disseminada pelo país. Concretamente, na região Alentejo, há 40 Centros, dos quais 62,5\% são promovidos por escolas públicas do Ministério da Educação. A importância deste dispositivo tem vindo a ser revelada no trabalho empírico de vários estudos (Esdime, 2007; Rico, 2007; Rico \& Libório, 2009; Nico, 2009, Nico, 2011) e projectos de investigação, como sejam a "Arqueologia das Aprendizagens no Concelho do Alandroal" (Nico et al, 2008) e "As Novas Núpcias da Qualificação no Alentejo", ambos projectos financiados pela Fundação para a Ciência e Tecnologia.

O estudo, que nos encontramos a desenvolver pode tornar-se um importante contributo para a avaliação do papel das diversas instituições na qualificação de um território/região e das pessoas que dele fazem parte, valorizando e integrando-as numa rede educativa. Esta rede deverá, na nossa opinião, estar evidenciada e valorizada nos instrumentos de planeamento e de ordenamento da rede educativa de um determinado território, sendo que estes estudos podem contribuir para a sua eventual melhoria e enriquecimento.

De acordo com um relatório da Direcção-Geral de Educação e Cultura da Comissão Europeia, intitulado Further measures to implement the action plan on adult learning: Updating the existing inventory on validation of non-formal and informal learning: Final report", Portugal encontra-se entre os cinco países (a par da Finlândia, França, Holanda e Noruega), que ocupam a mais elevada posição em matéria de validação das aprendizagens não formais e informais (www.anq.gov.pt).

\section{O contexto da intervenção (as instituições)}

A presente comunicação resulta de um projecto de investigação que se assumiu como uma investigação de cariz descritivo, com recurso a uma metodologia que contemplou abordagens quantitativas e qualitativas. 
As instituições seleccionadas e que foram objecto de análise nesta comunicação foram 4 Centros de Reconhecimento, Validação e Certificação de Competências a operar no Alentejo, em 2003:

1. ESDIME (Agência para o Desenvolvimento Local no Alentejo Sudoeste), com sede em Messejana (Aljustrel);

2. Fundação Alentejo, com sede em Évora;

3. Centro de Formação Profissional do IEFP, com sede em Portalegre;

4. ADL (Associação de Desenvolvimento do Litoral Alentejano), com sede em Santiago do Cacém;

\section{Caracterização dos indivíduos participantes no estudo}

Do total de 751 adultos certificados no ano de 2003, nos 4 Centros identificados, 206 responderam ao inquérito por questionário. A distribuição dos inquéritos, por Centro de RVCC (CRVCC), foi a seguinte:

Quadro $1-N .^{\circ}$ de respondentes

\begin{tabular}{|c|c|c|}
\hline Centro de RVCC & $\begin{array}{c}\mathbf{N}^{\mathbf{0}} \text { de indivíduos } \\
\text { certificados } \\
(\mathbf{2 0 0 3})\end{array}$ & $\begin{array}{c}\mathbf{N}^{\mathbf{0}} \text { de } \\
\text { respondentes }\end{array}$ \\
\hline Fundação Alentejo & 285 & 88 \\
\hline $\begin{array}{c}\text { ESDIME/ Agência para o } \\
\text { Desenvolvimento Local no Alentejo } \\
\text { Sudoeste }\end{array}$ & 360 & 84 \\
\hline $\begin{array}{c}\text { ADL/Associação Desenvolvimento } \\
\text { Litoral Alentejano }\end{array}$ & 70 & 24 \\
\hline $\begin{array}{c}\text { Centro de Formação Profissional } \\
\text { Portalegre }\end{array}$ & 36 & 10 \\
\hline Total & $\mathbf{7 5 1}$ & $\mathbf{2 0 6}$ \\
\hline
\end{tabular}

Da leitura do quadro anterior, podemos reter alguns aspectos que consideramos importantes:

- Aproximadamente $43 \%$ do total de respondentes pertencia ao CRVCC da Fundação Alentejo (42,7\%);

- $40,8 \%$ do total de respondentes pertencia ao CRVCC da ESDIME;

- O Centro onde se registou menor taxa de resposta (face ao . $^{\circ}$ total de adultos aí certificados) foi o Centro de RVCC da ESDIME (23,3\%);

- Com maior taxa de resposta ao inquérito (face ao n. ${ }^{\circ}$ total de adultos aí certificados), identificámos o Centro de RVCC da ADL (34,3\%). 
Quadro $2-N^{o}$ de respondentes por género

\begin{tabular}{|c|c|c|}
\hline Género & Frequência Absoluta (n. $\left.{ }^{\mathbf{*}}\right)$ & Frequência Relativa (\%) \\
\hline masculino & 89 & 43,20 \\
\hline feminino & $\mathbf{1 1 7}$ & $\mathbf{5 6 , 8 0}$ \\
\hline
\end{tabular}

Como se pode depreender da análise do Quadro 2, registou-se, na população respondente, uma predominância do género feminino.

Gráfico 1 -Adultos certificados por habilitações escolares de acesso ao processo (\%)

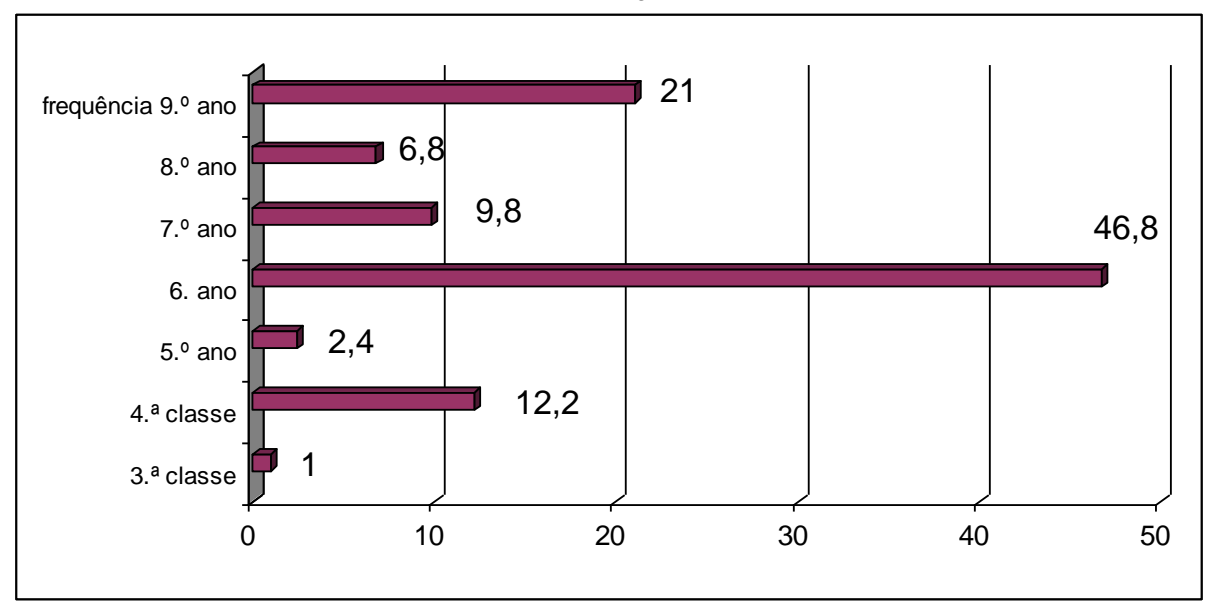

Em termos de habilitações escolares de acesso dos adultos certificados no ano 2003, verificámos o seguinte (cf. Gráfico 1):

- $46,8 \%$ tinha o $6 .^{\circ}$ ano de escolaridade (2. ${ }^{\circ}$ Ciclo do Ensino Básico);

- $21 \%$ dos adultos apresentava frequência de $9 .^{\circ}$ ano de escolaridade;

- $12,2 \%$ dos respondentes apresentou-se no CRVCC com a $4 .{ }^{a}$ classe $\left(1 .{ }^{\circ}\right.$ Ciclo do Ensino Básico).

- Apenas $1 \%$ dos adultos possuía a $3 .^{a}$ classe. Abaixo deste nível de escolaridade, não há registos.

Quantos aos níveis de certificação, os dados obtidos permitem-nos concluir que o maior n. ${ }^{\circ}$ de indivíduos certificou o nível B3 (3. ${ }^{\circ}$ Ciclo Ensino Básico).

Quadro 3 - Nível de certificação obtido

\begin{tabular}{|c|c|c|c|c|c|c|}
\hline \multirow{2}{*}{\multicolumn{2}{|c|}{ Género }} & \multicolumn{4}{|c|}{ Nível certificação } & \multirow[b]{2}{*}{ Total } \\
\hline & & $10^{\circ}$ Ciclo & 2. ${ }^{\circ}$ Ciclo & 3. ${ }^{\circ}$ Ciclo & Não respondeu & \\
\hline & masculino & 1 & 6 & 82 & 0 & 89 \\
\hline & feminino & 0 & 13 & 102 & 2 & 117 \\
\hline Total & & 1 & 19 & 184 & 2 & 206 \\
\hline
\end{tabular}




\section{Trajectória de aprendizagem e de prosseguimento de estudos dos adultos}

Através da aplicação do questionário aos indivíduos certificados no ano 2003, procurouse conhecer, entre outras dimensões, a trajectória de aprendizagem e de prosseguimento dos estudos dos indivíduos que concluíram, com sucesso, o processo de RVCC.

\subsection{O prosseguimento de estudos}

Dos 206 respondentes ao inquérito, apenas 15\% prosseguiu estudos após o processo de RVCC. Por cada indivíduo que prosseguiu os estudos, mais de 5 não o fizeram, como podemos observar a partir da leitura do quadro seguinte:

Quadro 4-Número de adultos que prosseguiu os estudos

\begin{tabular}{|c|c|c|c|}
\hline \multicolumn{2}{|c|}{} & Frequência Absoluta (n. ${ }^{\text {) }}$ & Frequência Relativa (\%) \\
\hline \multirow{3}{*}{} & Não & 175 & 85,0 \\
\cline { 2 - 4 } & Sim & $\mathbf{3 1}$ & $\mathbf{1 5 , 0}$ \\
\cline { 2 - 4 } & Total & $\mathbf{2 0 6}$ & $\mathbf{1 0 0 , 0}$ \\
\hline
\end{tabular}

Dos 31 adultos que prosseguiram estudos (15\% do total de respondentes), 3 concluíram o ensino secundário, e apenas 2 optaram por vias formativas para adultos, como os Cursos de Educação e Formação de Adultos (EFA). Apenas 1 adulto (3,2\%) prosseguiu estudos de nível superior. Destes 31 indivíduos, há uma predominância de mulheres que, após o processo de RVCC, prosseguiram os estudos (19).

No âmbito do Sistema RVCC, houve 1 adulto que referiu ter concluído o Ensino Básico, enquanto 2 já estavam a frequentar o RVCC de Nível Secundário.

Para os adultos que não tinham prosseguido estudos, procurámos saber se, efectivamente, pensavam vir a fazê-lo, no futuro. Verificamos que, num total de 175 respostas válidas, 134 adultos referiram que pretendiam prosseguir a sua aprendizagem em ambientes formais e certificados $(76,6 \%)$ :

Quadro 5 - Intenção de prosseguimento dos estudos

\begin{tabular}{|c|c|c|c|}
\hline \multicolumn{2}{|c|}{} & Frequência Absoluta (n. ${ }^{\mathbf{0}}$ & Frequência Relativa (\%) \\
\hline \multirow{3}{*}{ Sim } & $\mathbf{1 3 4}$ & $\mathbf{7 6 , 6}$ \\
\cline { 2 - 4 } & Não & 41 & 23,4 \\
\cline { 2 - 4 } & Total & $\mathbf{1 7 5}$ & $\mathbf{1 0 0 , 0}$ \\
\hline
\end{tabular}

Dos 134 adultos que manifestaram a vontade de continuar a estudar, as opções formativas indicadas radicaram em percursos de nível secundário: 39,6\% das escolhas (53 indivíduos) indicavam a conclusão do ensino secundário regular ("fazer o $12 .^{\circ}$ ano") e $\mathbf{3 8 , 1 \%}$ das 
escolhas (51 indivíduos) apontavam a eventualidade de se realizar o percurso de RVCC de Nível Secundário.

Os indivíduos do género feminino valorizaram mais o prosseguimento de estudos pois, dos 134 adultos, 79 são mulheres $(58,9 \%)$ e só 55 são homens, tal como se verificou anteriormente.

Quadro 6-Opções de prosseguimento dos estudos

\begin{tabular}{|c|c|c|}
\hline Opções identificadas pelos inquiridos & $\begin{array}{c}\text { Frequência } \\
\left.\text { Absoluta (n. }{ }^{\circ}\right)\end{array}$ & $\begin{array}{c}\text { Frequência } \\
\text { Relativa (\%) }\end{array}$ \\
\hline Fazer o $12 .^{\circ}$ ano & 53 & 39,6 \\
\hline RVCC NS & 51 & 38,1 \\
\hline Curso Profissional & 4 & 3,0 \\
\hline Curso Superior & 4 & 3,0 \\
\hline Cursos de actualização/aquisição competências & 2 & 1,5 \\
\hline $9 .^{\circ}$ ano & 1 & 0,7 \\
\hline Curso de Educação e Formação de Adultos & 1 & 0,7 \\
\hline RVCC NB & 1 & 0,7 \\
\hline Não identificou & 17 & 12,7 \\
\hline Total & 134 & 100,0 \\
\hline
\end{tabular}

Verificou-se a existência de um número, pouco significativo, de adultos que pretendia prosseguir estudos de nível secundário através da frequência de ofertas formativas e não apenas por um percurso de RVCC. São evidências desta conclusão, a fraca manifestação, à data, de interesse pelos Cursos Profissionais e Cursos EFA, com apenas 5 escolhas.

De referir a existência de adultos que, embora tenham prosseguido os estudos, manifestaram, no preenchimento do questionário, a sua vontade em continuar a estudar: um deles concluiu um Curso Profissional e pensava fazer um outro. Relativamente aos restantes, um deles frequentava um Curso Profissional e o outro o Ensino Secundário. Ambos pretendiam, no futuro, "fazer o 12. "ano".

Pareceu-nos existir a ideia pré-estabelecida e adquirida pelos adultos de que, para continuar a estudar, o melhor caminho seria o RVCC, o que não corresponde necessariamente à realidade. Um percurso de RVCC implica um acumular de experiência e de aprendizagem ao longo da vida, que só será possível de concretizar pela frequência de outras ofertas formativas e experiências tidas em contextos não formais e informais de aprendizagem, como por exemplo, os "cursos de actualização/aquisição de competências" referidos por 2 adultos.

Neste âmbito, as respostas aos questionários permitiram-nos também saber que $\mathbf{1 7}$ adultos já tinham realizado a sua inscrição no Centro para um novo percurso de RVCC de Nível 
Secundário. Refira-se que, no ano em que se aplicaram os questionários (2007), o processo de RVCC de nível secundário encontrava-se, ainda, na sua fase inicial de implementação.

De seguida, registamos alguns dos excertos dos questionários, no que a este aspecto diz respeito:

“(...) tenho aguardado com expectativa a chegada de nova oportunidade, para que isso seja possivel, já estou inscrita no mesmo centro (RVCC), espero conseguir (...)"

(Adulta certificada pelo Centro de RVCC da ESDIME)

"Fiz inscrição no 12. ${ }^{\circ}$ ano das Novas Oportunidades"

(Adulta certificada pelo Cento de RVCC da Fundação Alentejo)

Após identificarmos as opções individuais de cada adulto que pretendia prosseguir a sua qualificação (“o quê”), procurámos saber também quando é que essa intenção iria ocorrer (questão n. ${ }^{\circ} 19$ do Questionário), como a seguir se apresenta no Quadro 7:

Quadro 7 - Perspectivas temporais para o prosseguimento dos estudos

\begin{tabular}{|c|c|c|c|}
\hline \multicolumn{2}{|c|}{ Prosseguimento de estudos: quando? } & $\begin{array}{c}\text { Frequência Absoluta } \\
\left(\text { n. }^{\circ}\right)\end{array}$ & $\begin{array}{l}\text { Frequência } \\
\text { Relativa }(\%)\end{array}$ \\
\hline \multirow{4}{*}{ A curto prazo } & Brevemente & 14 & 10,4 \\
\hline & 2007 & 8 & 6,0 \\
\hline & 2008 & 4 & 3,0 \\
\hline & Sub-total & 26 & 19,4 \\
\hline \multirow{4}{*}{$\begin{array}{l}\text { A médio/ } \\
\text { longo prazo }\end{array}$} & Quando surgir a oportunidade & 38 & 28,4 \\
\hline & Quando for chamada pelo CRVCC & 9 & 6,7 \\
\hline & Quando tiver tempo disponível & 6 & 4,5 \\
\hline & Sub-total & 53 & 39,6 \\
\hline \multirow{2}{*}{ Não identifica } & Não sabe & 7 & 5,2 \\
\hline & Não respondeu & 48 & 35,8 \\
\hline & Sub-total & 55 & 41,0 \\
\hline \multicolumn{2}{|r|}{ Total } & 134 & 100,0 \\
\hline
\end{tabular}

Da leitura do Quadro 7, podemos concluir o seguinte:

- Dos 134 adultos que manifestaram a vontade de continuar a estudar, mais de $40 \%$ (55 adultos) não identificou o momento em que o pretendia fazer (não sabia ou não respondeu);

- É significativo o número de adultos (48) que não respondeu a este aspecto, o que poderá ser indício de alguma incerteza quanto ao futuro em matéria de formação e qualificação; 
- A médio/longo prazo, foi a resposta indicada por 53 dos inquiridos (39,6\% do total de indivíduos que responderam a este aspecto), os quais referiram que: "quando surgir a oportunidade" (28,4\%), ou "quando for chamada pelo CRVCC” $(6,7 \%)$. Seis indivíduos indicaram que esse acontecimento ocorrerá "quando tiver tempo disponivel" (4,5\%);

- A maior parte dos adultos, que mencionou ter vontade em continuar a estudar, perspectiva essa situação num horizonte temporal definido como a médio/longo prazo.

\section{Conclusões}

Concluindo, ao nível da trajectória de aprendizagem e de prosseguimento de estudos dos adultos, dos 206 indivíduos certificados no ano de 2003, nos 4 Centros de RVCC, em funcionamento na região Alentejo, 15,0\% dos inquiridos continuou a estudar e a apostar no seu percurso de formação e qualificação, com um maior número de mulheres a tomar essa opção.

Apesar de reconheceram mais dificuldades, na realização do processo de RVCC (Nico, 2009), verifica-se que são as mulheres as que mais apostam na continuidade dos estudos. Se, por um lado, uma minoria dos inquiridos prosseguiu estudos, por outro lado, verificamos que uma maioria perspectivava essa situação, a médio/longo prazo, o que demonstra um certo envolvimento e motivação por parte dos adultos certificados inquiridos, como se pode verificar através das palavras escritas dos inquiridos:

\footnotetext{
"Este processo deu-me a oportunidade de prosseguir estudos que, de alguma maneira, não foi possível por vários motivos."

“Após concluir o processo pelo Centro RVCC, pretendi continuar a estudar e matriculei-me na Escola Secundária ...."

"No meu caso, fiquei mais entusiasmada para estudar, não tenho dúvidas de que foi um estímulo para continuar a estudar..."
}

\section{Referências Bibliográficas}

ALONSO, L., et al (2001). Lista de competências-chave - Educação e Formação de Adultos. Lisboa: ANEFA.

ANEFA (2001). Conferência Europeia Educação e Formação de Adultos na Europa. As Competências-Chave para a Cidadania e Empregabilidade. Lisboa: ANEFA. 
CORREIA, A. (2006). "O Sistema Nacional de Reconhecimento, Validação e Certificação de Competências". In Albano Estrela et al (orgs). Actas do XVII Colóquio ADMEE-Europa. Lisboa: FPCE-UL. pp: 267-274.

DELORS, Jacques et al (1996). Educação: O tesouro a descobrir. Relatório da Comissão Internacional sobre Educação para o século XXI. Paris: Unesco / Rio Tinto: ASA.

ESDIME (2007). Estudo sobre o Impacto da Certificação de Competências na Vida das Pessoas: A Experiência da Esdime. Camarate: IEFP.

GOMES, M. \& SIMÕES, M. (2007). Carta de Qualidade dos Centros Novas Oportunidades. Lisboa: Agência Nacional para a Qualificação, I.P.

NICO, B., et al (2008). "Reconhecimento, Validação e Certificação de Competências no Alentejo: uma visão da realidade ". In Bravo Nico et al (Orgs). Aprender no Alentejo - IV Encontro Regional de Educação. Évora: Departamento de Pedagogia e Educação da Universidade de Évora. pp. 211-218

NICO, L. (2009). Avaliação do(s) Impacto(s) do Processo de Reconhecimento, Validação e Certificação de Competências (RVCC), no Alentejo (período 2001-2005) [Tese apresentada à Universidade de Évora tendo em vista a obtenção do Grau de Doutor em Ciências da Educação]. Évora: Universidade de Évora (policopiada).

NICO, L. (2011). A Escola da Vida: Reconhecimento dos Adquiridos Experienciais em Portugal. Fragmentos de Uma Década (2000-2010). Mangualde: Edições Pedago.

PIRES, A. (2005). Educação e Formação ao Longo da Vida: análise crítica dos sistemas e dispositivos de reconhecimento e validação de aprendizagens e competências. Lisboa: Fundação Calouste Gulbenkian e Fundação para a Ciência e Tecnologia.

RICO, H. (2007). O Impacto do Processo de RVCC: perspectivas dos adultos certificados. [Dissertação apresentada à Universidade de Coimbra, tendo em vista a obtenção do grau de Mestre em Ciências da Educação]. Coimbra: Faculdade de Psicologia e de Ciências da Educação da Universidade de Coimbra.

RICO, H. \& LIBÓRIO, T. (2009). Impacte do Centro de RVCC da Fundação Alentejo na qualificação dos alentejanos. Évora: Fundação Alentejo.

TRIGO, M. (2002). "O Sistema de Reconhecimento, Validação e Certificação de Competências". In Isabel Silva et al (Orgs). Educação e Formação de Adultos. Factor de Desenvolvimento, Inovação e Competitividade. Lisboa: ANEFA. pp. 54-71.

\section{Sítios de internet consultados}

www.novasoportunidades.gov.pt (acedido em 07 de Julho de 2011)

www.anq.gov.pt (acedido em 07 de Julho de 2011)

\section{Legislação referenciada}

Decreto-Lei n. ${ }^{\circ}$ 396/2007, de 31 de Dezembro 